\title{
Sefferson. Oculocardiac Reflex Elicited During Debridement of an Empty Orbit
}

James C. Tsai, MDa, James W. Heitz, MDb', Edward H. Bedrossian Jr., MD FACS ${ }^{c}$

${ }^{a}$ Thomas Jefferson University Hospital, 'befferson Medical College, Thomas Jefferson University, 'Wills Eye Institute

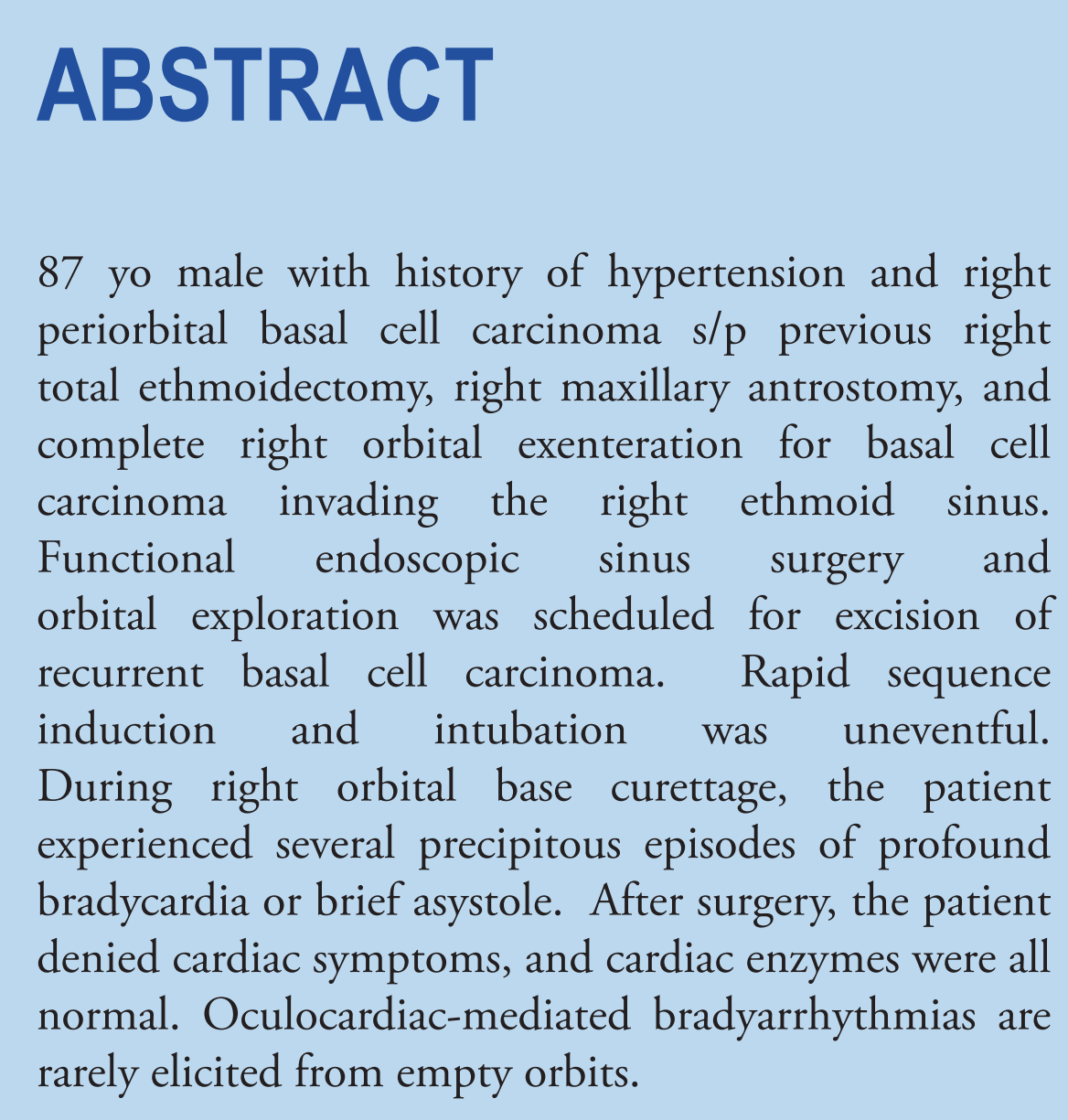

\section{INTRODUCTION:}

The oculocardiac reflex (OCR) is a well-described cardiac depressor reflex that may result in significant cardiac rate block or asystole. It is also known by the e, han Aschner-Dagnini Reflex, being independently reported in 1908 by both GuiseppeDagniniand later Bernard Aschner 12 The OCR is classically depicted as being precipitated by traction on the extraocular muscles or pressure on the globe. The ophthalmic branch of the trigeminal nerve serves par the efferent pathay We report the OCR resulting in episodes of manipulation within a previously enucleated empty orbit.

\section{CASE REPORT}

The patient was an 87 -year-old man $(61-\mathrm{kg}, 165-\mathrm{cm})$ with history of hypertension, gastro-esophageal reflux disease, cell carcinoma of skin with invasion of the ethmoid sinuses. The patient had a complete right orbital exenteration, right total ethmoidectomy, and right maxillary antrostomy one year prior. The patient presented for functional endoscopic sinus surgery and right orbital exploration for excision of basal cell carcinoma invading into the ipsilateral ethmoid sinus. His medications included omeprazole, levolhyrox-

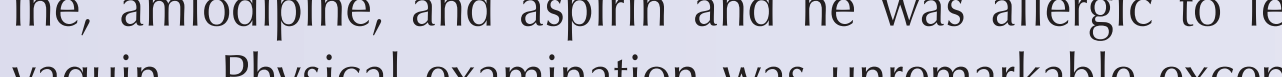
for the post-surgical changes of the head. A preoperative electrocardiogram (ECG) was normal with a sinus rate of 70 beats per minute (bpm)

Bag-mask ventilation was anticipated to be impossible due to the right maxillary antrostomy; consequently rapid sequence induction of general anesthesia was performed with fentanyl, lidocaine, propofol, and succinylcholine. The trachea was intubated and anesthesia was maintained with sevoflurane. The heart rate remained stable at around 70 bpm until approximately one hour into the procedure when a brief episode of asystole was noted for approximately 5 proximity to the stump of the optic nerve (figure 1 ). Normal sinus rhythm was restored without intervention, but bradycardia at approximately $20 \mathrm{bpm}$ recurred associated with curettage of the orbital base. The heart rate again returned to $60 \mathrm{bpm}$ without intervention. Another episode of bradycardia to approximately $20 \mathrm{bpm}$ was observed a few minutes later temporally-associated with additional curettage at the base of the right orbit. OCR was suspected as the cause of the bradycardia so the aneshelic depthwas increased to attenuate he relex, rrom Traction upon the optic nerve stump was still able provoke an abrupt and transient $10 \mathrm{bpm}$ deceleration of the heart rate despite the increased depth of anesthesia. The remainder of the procedure including emergence from general anesthesia and tracheal extubation were unremarkable. After surgery, the patient denied cardiac symptoms. Three sets of cardiac enzymes were negatice,

\section{DISCUSSION}

The oculocardiac reflex (OCR) is a well-described cardiac depressor reflex that may result in significant cardiac rate or rhythm changes including sinus and junctional bradycardia, heart block or asystole. ${ }^{3}$ Therefore, OCR can have profound hemodynamic consequences resulting in morbidity or mortality. ${ }^{4.5}$ The OCR is classically depicted as being precipitated by traction on the extraocular muscles or pressure on the globe. The stretch receptors in these

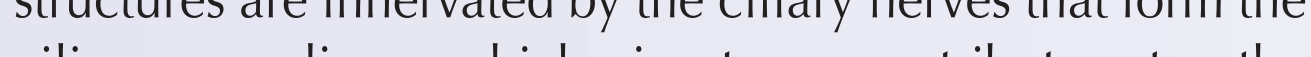
ciliary ganglion, which in turn contributes to the pathways converge in the sensory nucleus of the trigeminat nerve. The efferent pathway of the OCR is mediated by the motor nucleus of the vagus nerve resulting in the negative chronotropic and dysrhythmic effects on the heart (figure 2). ${ }^{6}$ A similar reflex has been reported without the prerequisite manipulation of areas innervated by the ophthalmic may be seen during maxillary osteotomy or maxillary advancement during Le Fort I osteotomy, soft tissue manipulation near the mandible, and procedures near the skull base The OCR may be a subset of a mare

We report the OCR resulting in episodes of profound badycardia and bief asystole during manipulation within numerous on the eye where orbital structures are intact, but only one prior report of the OCR during a procedure involving a previously enucleated orbit to our knowledge."1 OCR from direct manipulation of the optic nerve is an unusual feature of this report, but it is consistent with reports of OCR precipitated by optic nerve clamping during The occurrence of the OCR in the empty orbit demonstrates that orbital structures containing stretch receptors are not required for initiation of the OCR. subseque, palients remain a risk for OCR during enucleation or during fitting of an ocular prosthesis.

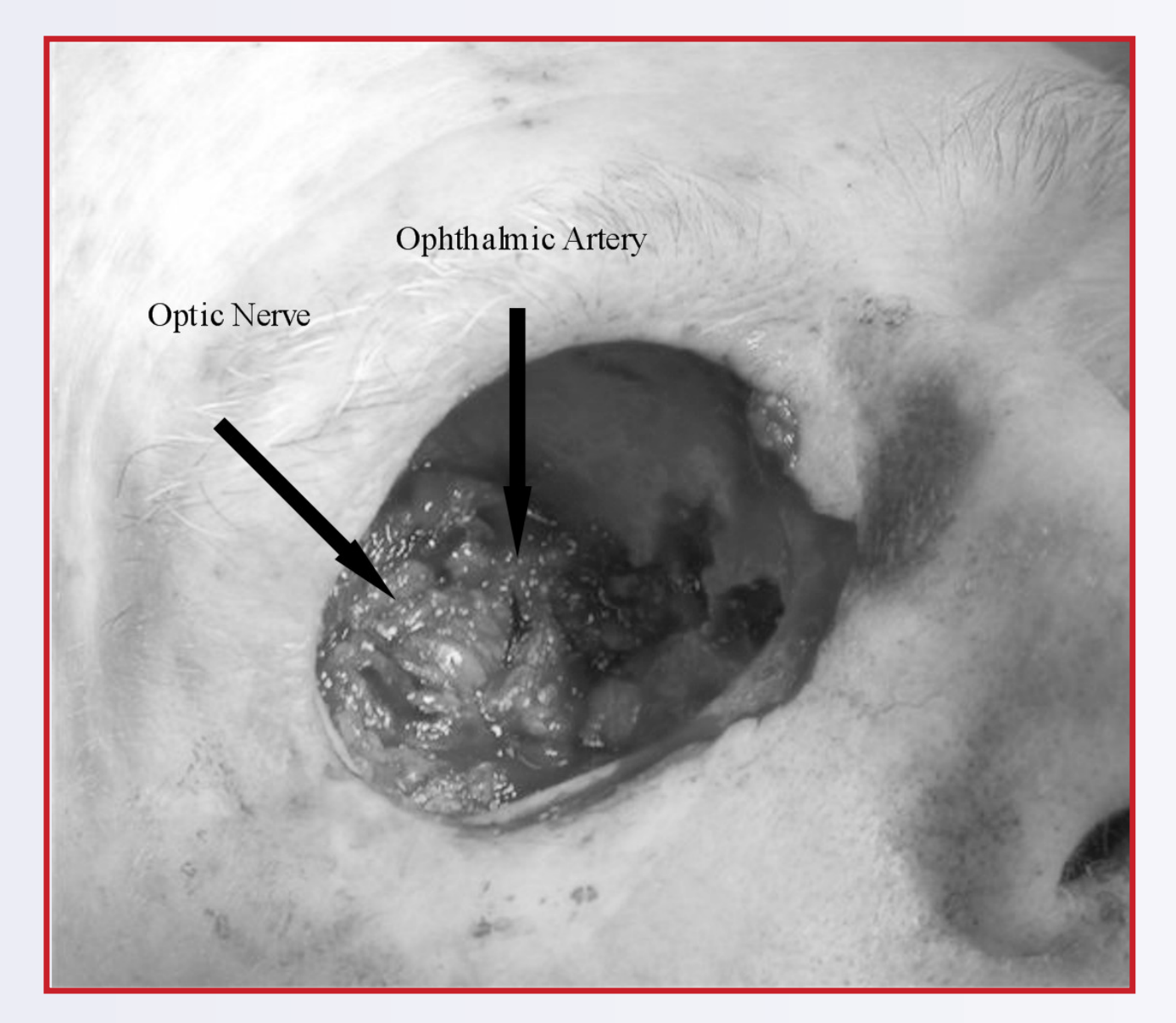

Figure 1: View of the empty orbit, the stump of the optic nerve and a suture on the ophthalmic artery are visible.

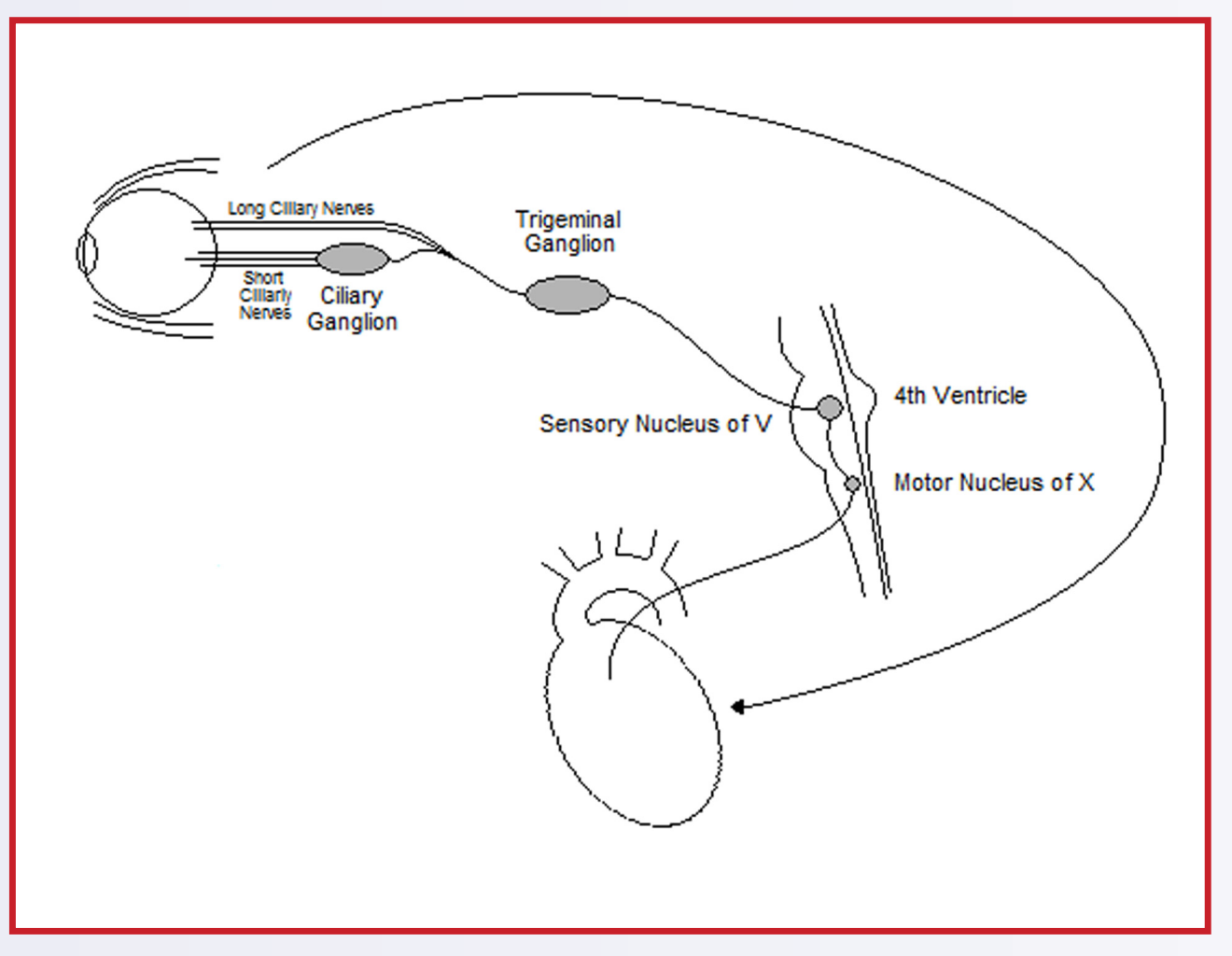

Figure 2: Oculocardiac Reflex Pathway
REFERENCES

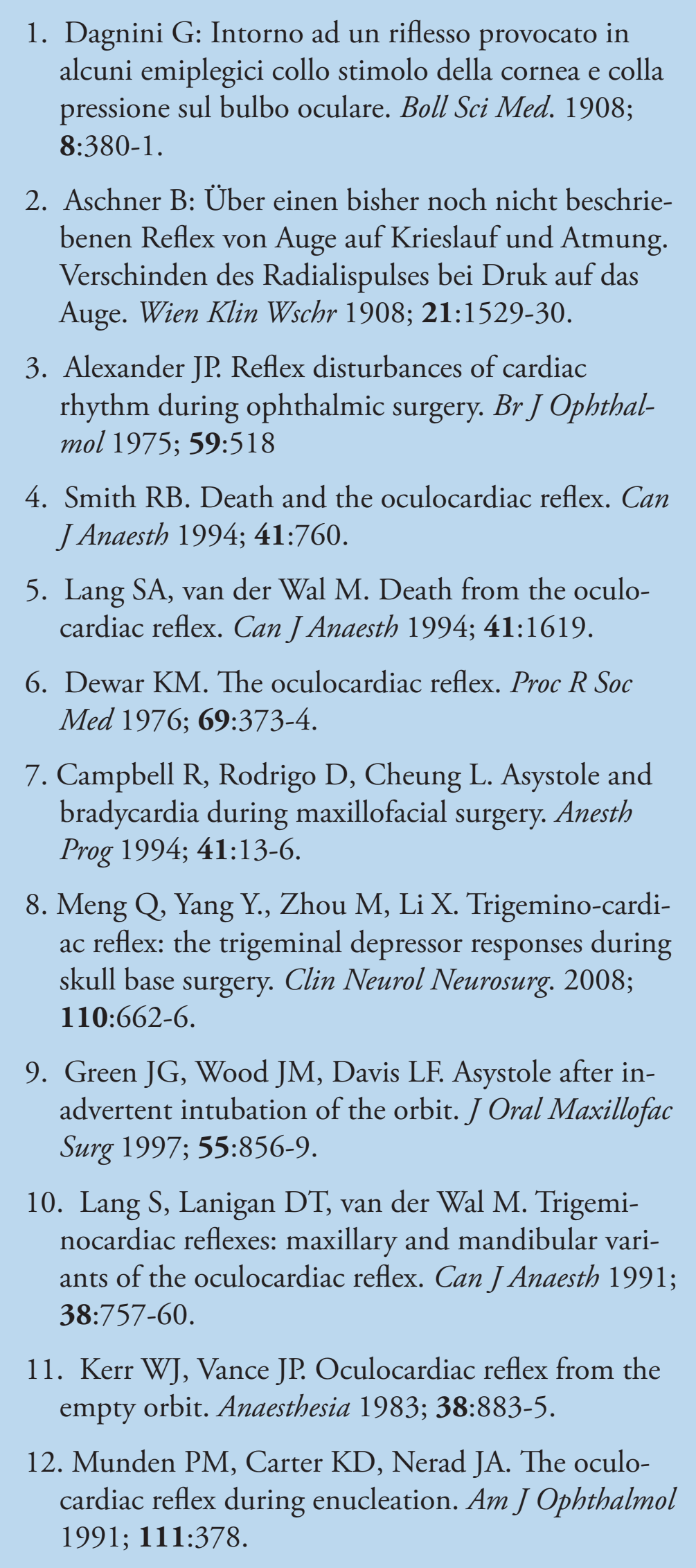

